SUPPORTING INFORMATION for:

\title{
Mass + retention time $=$ structure: A strategy for the analysis of $N$-glycans by carbon LC-ESI-MS and its application to fibrin $N$-glycans
}

\author{
Martin Pabst ${ }^{1}$, Jayakumar Singh Bondili ${ }^{1}$, Johannes Stadlmann ${ }^{1}$, Lukas Mach ${ }^{2}$, Friedrich \\ Altmann ${ }^{1}$
}

Biochemistry Division of the Department of Chemistry and ${ }^{2}$ Institute of Applied Genetics and Cell Biology, University of Natural Resources and Applied Life Sciences (BOKU), Vienna, Austria

\begin{abstract}
SI
The first three chapters describe the preparation or sources of the relevant glycosyltransferases and glycosidases and the preparation of the various glycan standards by the help of these enzymes and are as such part of the Experimental section.

The following chapter S-3.1. describes various observations leading to the choice of the particular LC-ESI conditions used for this study. Chapter S-3.2 describes details of the standard preparation and assignments. Chapter S-3.5 finally discusses aspects of in-source fragmentation, which becomes obvious with the use known standard compounds.
\end{abstract}

\section{Ad Experimental section}

\section{S-2.1 Glycoproteins and $N$-glycan preparation}

Human fibrinogen was a kind gift from Dr. Alfred Weber (Baxter BioScience, Vienna, Austria). Fibrinogens from cat and dog were purchased from Sigma-Aldrich (Vienna, Austria). Fibrins from cow, pig, mouse, Chinese hamster, horse and chicken were prepared from fresh blood, which was mixed $9: 1$ with $3.8 \%$ trisodium citrate (buffered to $\mathrm{pH} 7.4$ with $\mathrm{HCl}$; plus $0.02 \% \mathrm{NaN}_{3}$ ) to prevent clotting. Blood cells were removed by centrifugation at $1500 \times \mathrm{g}$. Clotting was initiated by addition of 0.05 volumes of $1 \mathrm{M} \mathrm{CaCl}_{2}$. The pellet was washed twice with $0.9 \% \mathrm{NaCl}$ and twice with water and freeze dried.

Erypo (Janssen-Silag Pharma, Vienna, Austria) was the source of recombinant erythropoietin (EPO). Bovine fetuin was obtained from Sigma-Aldrich.

Aliquots of ca. $10 \mu \mathrm{g}$ of each fibrin were dissolved in SDS-PAGE sample buffer and applied to reducing SDSPAGE, followed by staining with Coomassie Blue. Contact with acidic solutions was kept as short as possible to minimize loss of neuraminic acids. The bands comprising the not or hardly resolved $\beta$ and $\gamma$-chains were excised, carboxymethylated and digested with trypsin ${ }^{1}$. $N$-glycans were released by digestion with $0.6 \mu \mathrm{U}$ of peptide $N$ glycosidase $\mathrm{A}^{2}$. Oligosaccharides were isolated using carbon tips (Thermo Electron, Vienna, Austria) ${ }^{3,4}$. A large scale preparation of bovine fibrin glycans was undertaken with peptic glycopeptides, which were purified and digested with peptide $N$-glycosidase $\mathrm{A}^{5}$. Likewise, Man5 oligosaccharides were obtained by digestion of taka-amylase peptides ${ }^{5}$. 
Internal standards were prepared by digestion of human apo-transferrin with endo- $N$-acetylglucosaminidase F2 and of bovine fetuin with endo- $N$-acetylglucosaminidase F3 (Merck Biosciences, Darmstadt, Germany). The resulting mixture of $\mathrm{Na}^{6-4} \mathrm{Na}^{6-4}-E$ and $\mathrm{Na}^{3-4} \mathrm{Na}^{6-4}-E$ (the $E$ indicating the endo-glycosidase cleavage) and triantennary glycans was used without reduction and gave four peaks of $m / z=1010.9$ for the doubly charged ions.

\section{S-2.2 Glycosyltransferases and glycosidases}

$\alpha 2,3-$ and $\alpha 2,6-$ Sialyltransferase (SiaT3Gal; EC 2.4.99.and SiaT6Gal; EC 2.4.99.) were purchased from

Calbiochem. $\beta 1,4$-galactosyltransferase from bovine milk was obtained from Sigma-Aldrich. Recombinant $\alpha 1,3-$ galactosyltransferase was bought from Fluka. Recombinant rabbit GlcNAc-transferase I was available from a previous study ${ }^{6}$.

Recombinant mouse $\beta 1,3$-galactosyltransferase 1 and human GlcNAc-transferase III were produced in insect cells. Briefly, a cDNA fragment encoding a soluble form of mouse $\beta 1,3$-galactosyltransferase 1 encompassing amino acids 34-326 (UniProt O54904) was generated by PCR using the primer pair 5'-

TATTCTGCAGGGCTCCAAGCCGTTCAGTCACC-3' (forward) and 5'-

TATTGAATTCCTAACATCTCAGATGCTTCTTG-3' (reverse), and a plasmid containing the complete $\beta 1,3-$ galactosyltransferase 1 open reading frame ${ }^{7}$ as template. The PCR product was digested with the restriction enzymes PstI and EcoRI at the underlined sites and ligated into pVTBacHis-1 baculovirus transfer vector ${ }^{8}$ treated with the same enzymes. In the case of human GlcNAc-transferase III, total RNA was extracted from HepG2 human hepatoma cells using the TRIzol reagent (Invitrogen, Lofer, Austria). First-strand cDNA was synthesized from $5 \mu \mathrm{g}$ total RNA using Superscript III reverse transcriptase (Invitrogen) and an oligo(dT) primer. The coding sequence for a soluble form of human GlcNAc-transferase III consisting of amino acids 23531 (GenBank accession No. NM_002409) was then amplified with the primer combination 5'-

CGGGATCCCTTCTTCAAGACCCTGTCCTATGTCA-3' (forward) and 5'-

GGAATTCCCTAGACTTCCGCCTCGTCCAGTT-3' (reverse) using the GC-RICH PCR kit (Roche, Vienna, Austria). The PCR product thus obtained was cleaved with BamHI and EcoRI at the underlined sites and ligated into BamHI/EcoRI-digested pVT BacHis1. Expression of the two transferases in S. frugiperda Sf21 cells was performed exactly as described ${ }^{9}$. Culture supernatants of baculovirus-infected cells were then tested for the respective enzymatic activity. Activity assays were done in $0.1 \mathrm{M} 2-(\mathrm{N}$-morpholino) ethanesulfonic acid buffer (pH 6.8) containing $10 \mathrm{mM} \mathrm{MnCl}_{2}$, using $50 \mu \mathrm{M}$ dabsylated GnGn-tetrapeptide and either $1 \mathrm{mM}$ UDP-galactose or $1 \mathrm{mM}$ UDP-GlcNAc as substrates. Conversion of dabsylated GnGn-tetrapeptide into the respective product was monitored by MALDI-TOF-MS as described ${ }^{10}$.

The galactosidase from Aspergillus oryzae, which is essentially $\beta 1,4$-specific, was purified as described ${ }^{11}$. The $\beta 1,3$-preferring galactosidase from Xanthomonas manihotis was purchased as a recombinant enzyme from New England Biolabs (Ipswich, MA, USA). Jack bean $\alpha$-mannosidase and bovine kidney $\alpha$-fucosidase were obtained from Sigma-Aldrich. All digestions with exoglycosidases were performed in $50 \mathrm{mM}$ sodium citrate buffer, $\mathrm{pH}$ 4.5 . 


\section{S-2.3 Biosynthesis of glycan structures}

Various diantennary $\mathrm{N}$-glycans were prepared from $\mathrm{A}^{4} \mathrm{~A}^{4}$, which is the major structure of chemically desialylated bovine fibrin $N$-glycans. The three additional peaks containing $\beta 1,3$-linked galactose (see also section 3.2) were separated by PGC-HPLC with UV detection at $210 \mathrm{~nm}$ on a $150 \times 3$ column at flow rate of 0.6 using the solvents described in section 2.4. The four diantennary glycans (Fig. 2) were incubated with a limiting amount of $\alpha 2,3-$ sialyltransferase to yield a mixture of mono- and di- $\alpha 2,3$-sialylated glycans. Analogously, $\alpha 2,6$-sialyltransferase was used to generate mono- and di- $\alpha 2,6$-sialylated glycans. In several cases, mixtures of isomers were formed where the exact assignment of the resulting peaks was not possible at first. As some fibrin samples contained certain isomers in very dissimilar amounts, an assignment could be made by successive exo-glycosidase digestions, which are described in the Results section.

Finally, the two sialyltransferases were also applied consecutively to build disialylated glycans with mixed sialylation. To counteract the pronounced branch preference of both sialyltransferases, the whole procedure was performed twice, once with SiaT3Gal and once with SiaT6Gal as the first enzyme. The two preparations were mixed to obtain balanced amounts of $\mathrm{Na}^{6-4} \mathrm{Na}^{3-4}$ and $\mathrm{Na}^{3-4} \mathrm{Na}^{6-4}$. For the glycans with one 3-linked Gal, SiaT6Gal was used first.

Three more exotic combinations of five hexose and four hexosamine residues were prepared from Man5, which was isolated from fungal amylase ${ }^{12}$ and incubated with recombinant GlcNAc-transferase I to yield Man5Gn. From this intermediate, Man5An ( $\mathrm{Man}_{5} \mathrm{GalNAcGlcNAc}_{3}$ ) was generated by $\beta 1$,4-galactosyltransferase in the presence of UDP-GalNAc. Another route made use of recombinant GlcNAc-transferase III leading at first to Man5Gnbi ( $\left(\mathrm{Man}_{5} \mathrm{GlcNAc}_{4}\right)$. This isomer was further modified to Man4A ${ }^{4}$ bi $\left(\mathrm{Man}_{4} \mathrm{GalGlcNAc}_{4}\right)$ by $\beta 1,4-$ galactosyltransferase in the presence of UDP-Gal and jack bean $\alpha$-mannosidase, which can be assumed to remove the $\alpha 1$,6-linked mannose first ${ }^{13}$. The type 1 chain (containing $\beta 1,3$-linked Gal) was not investigated in the hybrid-type glycans.

For the assignment of branch isomers, the reference compounds MGn and GnM were generated from GnGn by limited digestion with $N$-acetylglucosaminidase. MGn was additionally prepared by $\alpha$-mannosidase treatment of Man5Gn.

Glycans were desialylated by incubation in $2 \mathrm{M}$ acetic acid at $80^{\circ} \mathrm{C}$ for $2 \mathrm{~h}$. 


\section{Ad Results section}

\section{S-3.1 An LC-ESI-MS system for native glycans}

We set out to separate glycoprotein $N$-glycans by PGC chromatography using conditions successfully applied in several recent investigations (i.e. acetonitrile in $5 \mathrm{mM}$ ammonium acetate, $\mathrm{pH}$ 9.6) in conjunction with negativeion ESI-MS detection ${ }^{14-16}$. This was an obvious choice because we aimed at analysing neutral as well as multiply sialylated and even sulfated $N$-glycans. However, the results obtained with samples prepared from bovine fetuin (mainly trisialylated glycans) ${ }^{17}$, or EPO (mainly tetrasialylated structures) ${ }^{18}$ were frustrating as we could not find the multiply sialylated species. Examination of the samples by MALDI-MS in the negative mode or, after carboxyl methylation, in the positive mode, confirmed the presence of tri- and tetrasialylated glycans in our samples, but they did not appear in the LC-ESI-MS data. A clue came from the observation that even glycans with only two sialic acids eluted quite late and gave - unlike neutral glycans - very broad peaks. Although this observation contrasted with some recent papers ${ }^{14,16,19}$ it matched several reports where only $N$-glycans with two sialic acids at the most were found ${ }^{14,15,20}$.!

To facilitate elution of sialylated N-glycans in our LC-MS system, we experimented with different pH-values. The solvent system for peptides, i.e. $0.1 \%$ formic acid gave the same unsatisfactory result as the alkaline solvents. However, buffering of the formic acid significantly affected the elution behaviour of sialylated Nglycans and led to the facile elution even of tetrasialylated N-glycans with $\alpha 2,3$-linkages - an especially late eluting glycan structure (Fig. S-1). Under these conditions, apparently all N-glycans from EPO could be recovered as indicated by a comparison of peak heights of the sum spectrum from the PGC-LC-MS run with that of normal-phase HPLC of flourescence labelled glycans (Fig. S-1). Noteworthy, this chromatogram was acquired in the positive mode. Although one would assume that ionization of sialylated glycans were more intense in the negative mode, off-line experiments with a neutral $\mathrm{N}$-glycan $\left(\mathrm{A}^{4} \mathrm{~A}^{4}\right)$ and a monosialylated $N$ glycan $\left(\mathrm{A}^{4} \mathrm{Na}^{6-4}\right)$ led to the surprising conclusion that the yield of positive and negative ions is rather constant over a wide $\mathrm{pH}$ range (Fig. S-2). For tri- and tetrasialylated glycans only the relative, but not the absolute sensitivity is higher in the negative mode under our chromatographic conditions (data not shown).

Initial trials with ammonium formiate buffer of $\mathrm{pH} 3.0$ and disialylated N-glycans with different sialic acid linkages showed a very promising selectivity of the PGC column. Experiments with PA-labelled disialo Nglycans and fluorescence detection indicated that this isomer selectivity was highest at slightly acidic $\mathrm{pH}$ and detiorated at neutral and alkaline $\mathrm{pH}$ values (data not shown). As negative mode detection offered no profound advantage, we performed all the experiments described below in the positive mode, which provides a more even ionization intensity over the acetonitrile concentration range relevant for PGC-LC (Fig. S-2). Preliminary experiments suggested that the system can also be operated in the negative ion mode, which might be preferable for sulfated glycans (data not shown).

To prevent complication of chromatograms by the anomeric forms of reducing sugars, the standard protocol includes reduction of the glycans by sodium borohydride, which is decomposed to borate before chromatography. 


\section{S-3.2 Building a biosynthetic retention time library for diantennary $\mathrm{N}$-glycans}

\section{Non-sialylated diantennary structures}

The four isomers of an asialo-diantennary glycan, $A^{4} A^{4}, A^{4} A^{3,} A^{3} A^{4}$ and $A^{3} A^{3}$ (Fig. 1A) were prepared in two ways. Preparative amounts of these N-glycans were obtained from bovine fibrin. The partial resistance of bovine fibrin towards fungal $\beta$-galactosidase indicated these fractions to contain $\beta 1,3$-linked $\mathrm{Gal}^{11}$. Also, a glycopeptide fraction from bovine fibrin enriched in $\beta 3$-galactosylated structures has recently been used as a substrate for tomato $\alpha 1,4$-fucosyltransferase ${ }^{21}$. We have fractionated unlabelled, reduced oligosaccharides on a PGC column with UV detection. A similar pattern was obtained with capillary LC-ESI-MS (Fig.3B). To substantiate that the minor peaks in bovine fibrin contain $\beta 1,3$-galactose, GnGn-PA was incubated with recombinant mouse $\beta 1,3-$ galactosyltransferase to yield $\mathrm{A}^{3} \mathrm{~A}^{3}, \mathrm{~A}^{3} \mathrm{Gn}$ and $\mathrm{GnA}^{3}$. The retention times of these products coincided with those of the bovine fibrin $N$-glycans insensitive to $\beta 1$,4-galactosidase treatment. $\beta 1,3$-linked Gal conferred a small increase in retention of PA-glycans on the PGC column. The effect impinged differently depending on the branch that was $\beta 1,3$-galactosylated (data not shown).

The four AA isomers in the fractions 1 to 4 were identified by exoglycosidase digestions as $\mathrm{A}^{4} \mathrm{~A}^{4}, \mathrm{~A}^{4} \mathrm{~A}^{3}$, $A^{3} A^{4}$ and $A^{3} A^{3}$, respectively (Fig. 2A). The identification of peaks 2 and 3 as $A^{4} A^{3}$ and $A^{3} A^{4}$, respectively, was achieved by removal of the 4-linked Gal residues by fungal galactosidase and subsequent digestion with $\beta$ - $N$ acetylglucosaminidase to obtain either $\mathrm{MA}^{3}$ or $\mathrm{A}^{3} \mathrm{M}$. Upon heat inactivation of the glycosidases present, the glycans were converted by Xanthomonas $\beta$-galactosidase to $\mathrm{MM}$ or $\mathrm{GnM}$, for which reference compounds were available from previous studies ${ }^{12}$.

Theoretically, a few more structures of mass 1642.6 Da can occur as detailed in a later chapter.

\section{Monosialylated diantennary structures}

To prepare the large panel of sialylated isomers and to determine the retention times of the various possible mono- and disialylated $N$-glycans, we used the neutral acceptors depicted in Fig. 1A and recombinant sialyltransferases. Incomplete processing with SiaT6Gal produced a mixture of the isobaric pair $\mathrm{Na}^{6-4} \mathrm{~A}^{4}$ and $\mathrm{A}^{4} \mathrm{Na}^{6-4}$, of $\mathrm{Na}^{6-4} \mathrm{Na}^{6-4}$ and of residual $\mathrm{A}^{4} \mathrm{~A}^{4}$. The very different abundance of the two monosialylated isomers allowed branch assignment without preparative separation of the isomers. Similar as for the $\mathrm{A}^{3} \mathrm{~A}^{4}$ isomers, the glycans were treated with $\beta 1$,4-preferring galactosidase and $\beta$ - $N$-acetylglucosaminidase. The second antenna was then unveiled by chemical desialylation, which was followed by a second round of degalactosylation. Thus $\mathrm{A}^{4} \mathrm{Na}^{6-4}$ with the Neu5Ac located on the 3-arm was assigned as the major isomer, which is in line with the branch specificity of bovine colostrum $6-\mathrm{Sia}^{2} \mathrm{~T}^{22}$.

With $\mathrm{A}^{4} \mathrm{~A}^{3}$ the product of SiaT6Gal could only be $\mathrm{Na}^{6-4} \mathrm{~A}^{3}$, and with $\mathrm{A}^{3} \mathrm{~A}^{4}$ only $\mathrm{A}^{3} \mathrm{Na}^{6-4}$ because of the particular substrate specificity of the SiaT6Gal ${ }^{23}$. In contrast, SiaT3Gal generated two isomers from each AA species, i.e. a total of 8 isomers. One isomer strongly dominated and from the experiments with both sialyltransferases it became obvious that SiaT3Gal also preferred to decorate the 3-arm. The isomer pairs $\mathrm{Na}^{3-4} \mathrm{~A}^{3} / \mathrm{A}^{4} \mathrm{Na}^{3-3}$ and $\mathrm{Na}^{3-}$ ${ }^{3} \mathrm{~A}^{4} / \mathrm{A}^{3} \mathrm{Na}^{3-4}$ could be identified using the fungal $\beta$-galactosidase, which only degraded the respective isomer with the unsubstituted 4-linked Gal residues ${ }^{11}$. Finally, $\mathrm{A}^{3} \mathrm{~A}^{3}$ was incubated with $\mathrm{SiaT} 3 \mathrm{Gal}$ to yield $\mathrm{Na}^{3-3} \mathrm{~A}^{3}$ and $\mathrm{A}^{3} \mathrm{Na}^{3-3}$ (and $\mathrm{Na}^{3-3} \mathrm{Na}^{3-3}$, see below). Two peaks were obtained but not assigned to the individual isomers. The 
twelve isomers with the composition Neu5 $\mathrm{Ac}_{1} \mathrm{Hex}_{5} \mathrm{HexNAc}_{4}$ could all be separated with only $\mathrm{A}^{3} \mathrm{Na}^{6-4}$ and $\mathrm{A}^{3} \mathrm{Na}^{3-4}$ eluting very closely (Fig. 3A). Sialylated hybrid-type glycans have not yet been considered as no hint at the occurence of hybrid-type glycans in the fibrins was found.

\section{Disialylated diantennary structures}

Disialylated structures arose as side-products in the preparations shown in Fig. 1B. Preparation and assignment of the compounds with uniform sialyl linkage, i.e. $\mathrm{Na}^{6-4} \mathrm{Na}^{6-4}, \mathrm{Na}^{3-4} \mathrm{Na}^{3-4}, \mathrm{Na}^{3-3} \mathrm{Na}^{3-4}, \mathrm{Na}^{3-4} \mathrm{Na}^{3-3}$, and $\mathrm{Na}^{3-3} \mathrm{Na}^{3-3}$ was a relatively simple task. To obtain mixed sialylation modes, $\mathrm{A}^{4} \mathrm{~A}^{4}$ was at first partially 6-sialylated and then incubated with SiaT3Gal to yield the mixture $\mathrm{Na}^{6-4} \mathrm{Na}^{3-4}$ and $\mathrm{Na}^{3-4} \mathrm{Na}^{6-4}$ (besides some $\mathrm{Na}^{6-4} \mathrm{Na}^{6-4}$ and $\mathrm{Na}^{3-4} \mathrm{Na}^{3-4}$ ). However, because the two peaks arose in a ratio of ca. $1: 20$, the whole procedure was repeated with the two enzymes in reversed order. In this case, the other isomer was preferentially formed proving that both SiaT6Gal and SiaT3Gal prefer the 3-arm of diantennary substrates. Finally, the two substrates with 3-and 4-linked Gal were incubated with SiaT6Gal. The resulting products $\mathrm{Na}^{6-4} \mathrm{~A}^{3}$ and $\mathrm{A}^{3} \mathrm{Na}^{6-4}$ were 3-sialylated to obtain $\mathrm{Na}^{6-4} \mathrm{Na}^{3-}$ ${ }^{3}$ and $\mathrm{Na}^{3-3} \mathrm{Na}^{6-4}$. Thus, nine disialylated glycans of composition Neu5 $\mathrm{Ac}_{2} \mathrm{Hex}_{5} \mathrm{HexNAc}_{4}\left(\mathrm{~m} / \mathrm{z}\right.$ of $[\mathrm{M}+2 \mathrm{H}]^{2+}=$ 1113.4) were generated (Fig. 1B). All these nine isomers could be separated by the PGC capillary LC system (Fig. 3B).

\section{Hybrid-type and alpha-galactosylated N-glycans isobaric with diantennary glycans}

Taking into account hybrid-type glycans a few more isomers of a mass equal to that of the AA series can be constructed. One is Man5 $\mathrm{An}^{4}$, i.e. Man5Gn plus a GalNAc residue. The second is Man5Gnbi, i.e. Man5Gn with a bisecting GlcNAc (Fig. 1D). These two structures could be separated from the AA series (Fig. 2A). Given the strong effect of bisecting GlcNAc, the two other possible isobaric structures Man4 $\mathrm{A}^{4} \mathrm{bi}$ or Man $4 \mathrm{~A}^{3}$ bi presumably likewise elute ahead of $\mathrm{A}^{4} \mathrm{~A}^{4}$.

Another source of isobaric structures could be the action of $\alpha 1,3$-galactosyltranferase on diantennary glycans lacking one Gal residue (Fig. 2D). The isomers $\mathrm{GnA}^{\alpha 3-4} / \mathrm{A}^{\alpha 3-4} \mathrm{Gn}$ were synthesized from $\mathrm{A}^{4} \mathrm{~A}^{4}$ by partial, enzymatic addition of an $\alpha$-Gal residue and removal of the remaining free $\beta 1,4-G a l$. They eluted in the vicinity of $\mathrm{A}^{4} \mathrm{~A}^{3} / \mathrm{A}^{3} \mathrm{~A}^{4}$ but some separation was achieved even though the gradient was not optimized for these early eluting, neutral structures (Fig. 2). Structures containing both $\alpha 1,3-G a l$ and $\beta 1,3-G a l$ were not considered because $\alpha 1,3$-galactosyltransferase was found to act only slowly on type I chains, suggesting it to be outperformed by sialyltransferases in vivo. 


\section{S-3.5 Aspects of collision-induced and in-source fragmentation}

Fragment spectra of $\mathrm{Na}^{6-4} \mathrm{Na}^{6-4}$ and $\mathrm{Na}^{3-4} \mathrm{Na}^{3-4}$ were acquired off-line in positive mode to evaluate their usefulness for the structural assignments of peaks. Although subtle quantitative differences could be observed, we concluded that MS/MS could not contribute much to the information provided by LC-ESI-MS in the particular case of linkage isomers. For more complex samples, where complete separation of all isomers cannot be achieved, MS/MS may facilitate unambiguous results. With current technology, however, the semiquantitative information contained in peak areas is lost and the determination of correct retention times is impeded by the switching between operation modes. Therefore, the same sample would have to be run twice. Another issue is the in-source fragmentation of glycans, which is an unavoidable by-product of electrospray ionization. Remarkably, smaller glycans without sialic acid have a stronger spontaneous dissociation than large sialylated glycans, of which only a few percent underwent fragmentation (Fig. S-6). Use of pure standard substances allows the relevant fragments of a molecular ion and their relative height to be determined. Such knowledge is of importance for interpreting LC-MS runs of samples which contain sugars of widely differing mass. 
Figure S-1: Examples of diantennary Nglycans with different linkages of galactose and sialic acid in the classical style and in the cartoon depiction suggested by the Consortium of Functional glycobiology

(www.functionalglycomics.org). Dark squares stand for GlcNAc, white squares for GalNAc, grey circles for mannose, white circles for galactose and grey diamonds for sialic acid. The angles of lines depict the linkage as indicated. Structure names have been made according to the "proglycan" system (www.proglycan.com).

Figure S-2. pH and solvent dependence of signal intensity in positive and negative ESI-MS. Equal concentrations of a monosialylated $N$-glycan $\left(\mathrm{A}^{4} \mathrm{Na}^{6-4}\right)$ were introduced off-line with varying solvent compositions. Panel A shows the ionization efficiency over a broad range of acetonitrile concentration. The aequous component was either ammonium formiate buffer pH 3.0 (-ם-), pH 6.5 ($\diamond$-), and pH 9.0 (-०-), $65 \mathrm{mM}$ each. Dashed lines depict results for negative, solid lines for positive ion mode. The strong increase of negative ion intensity with higher acetonitrile concentrations is, however, accompanied by an equally strong increase of background signals. Panel $\mathbf{B}$ is a zoom for the solvent concentration range relevant for PGC-LS-ES-MS showing only the results for $\mathrm{pH}$ 3.0.
Neu5Ac $\alpha-6$ Gal $\beta-4 G I c N A c \beta-2 M a n \alpha$ ${ }_{3}^{6} \operatorname{Man} \beta-4 \mathrm{GlcNAc} \beta-4 \mathrm{GlcNAc} \beta-$

Neu5Ac $\alpha-6$ Gal $\beta-4 G I c N A c \beta-2 M a n \alpha^{\prime}$

$$
\mathrm{Na}^{6-4} \mathrm{Na}^{6-4}
$$

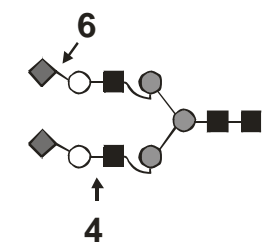

Neu5Ac $\alpha-3$ Gal $\beta-3$ GlcNAc $\beta-2$ Man $\alpha$ ${ }_{3}^{6} \operatorname{Man} \beta-4 \mathrm{GlcNAc} \beta-4 \mathrm{GlcNAc} \beta-$ Neu5Ac $\alpha-3$ Gal $\beta-3$ GIcNAc $\beta-2$ Man $\alpha$
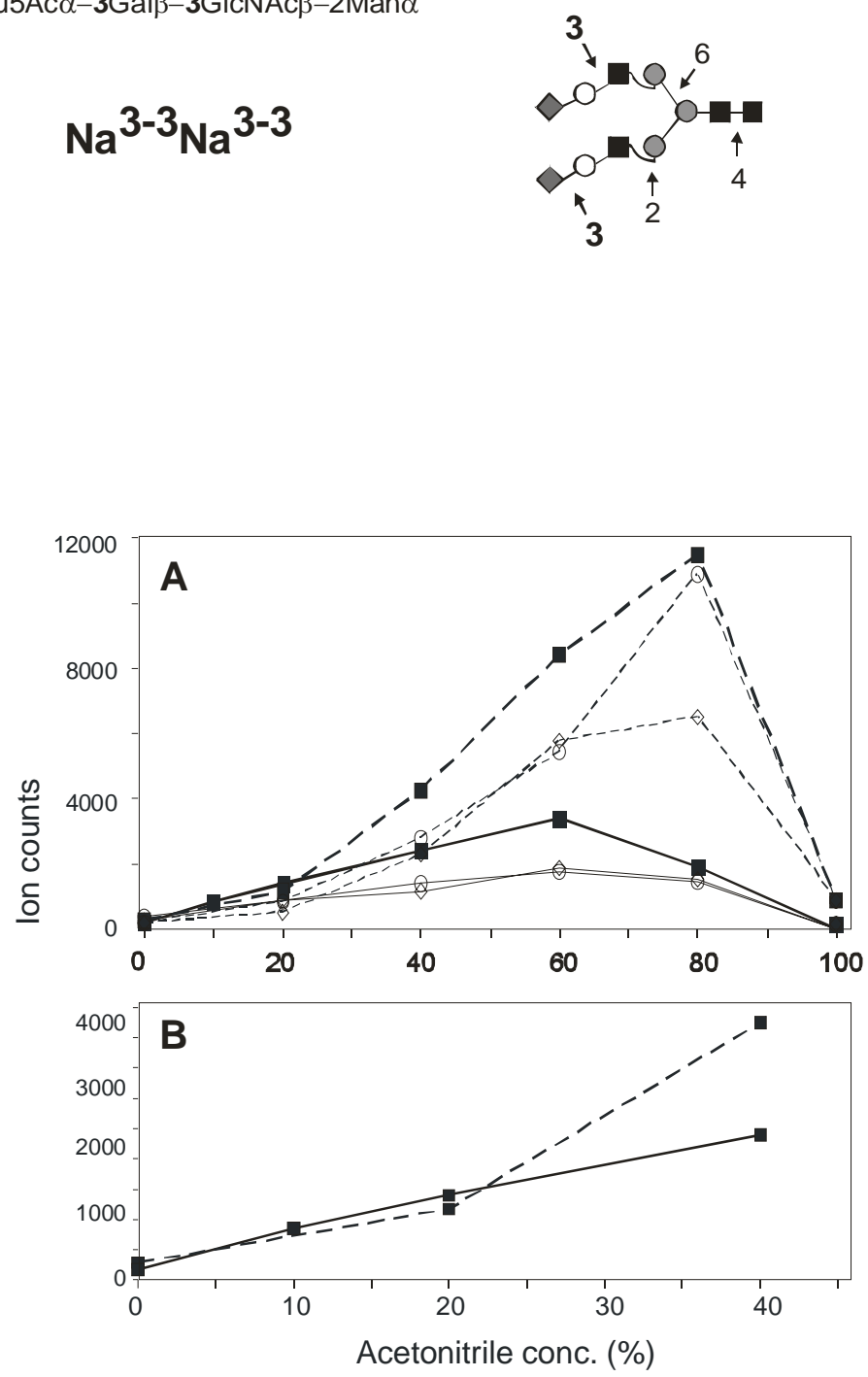
Figure S-3. PGC-LC-ESI-MS of $N$-glycans from recombinant human erythropoietin. Panel A shows a normal-phase HPLC chromatogram with fluorescence detection of 2-aminopyridine-labelled EPO- $N$-glycans. The major peaks were collected and identified by MALDI-TOF MS as consisting of glycans with 2 sialic acids, $2 \mathrm{~N}$-acetyllactosamine units and 1 fucose residue $=2$ S 2Ln F etc.. Panel B is the summed and deconvoluted spectrum of the entire elution range (70-95 $\mathrm{min}$ ) of EPO glycans. Without enlarging on the separation of isomers, the spectrum demonstrates that even tetrasialylated glycans can be detected in the positive ion mode.

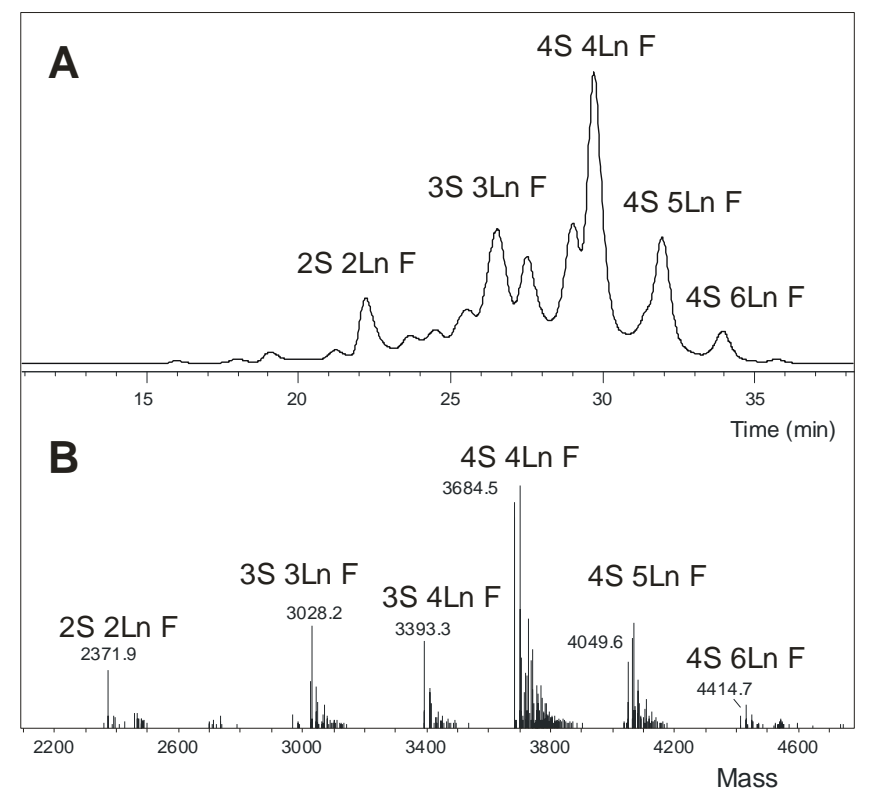

Figure S-4. Presence of N-glycolyl-neuraminic in bovine fibrin glycans. The chromatograms are from the PGCLC-ESI-MS analysis of reduced N-glycans from bovine fibrin (as in Fig. 7). In addition to the SIM trace for glycans with only Neu5Ac (0 Gc), those for glycans with 1 Neu5Gc (1 Gc) and 2 Neu5Gc residues (2 Gc) are shown. The SIM masses for the traces 0 Gc, 1 Gc and 2 Gc were 1113.9. 1121.9 and 1129.9, respectively. Oxidation of the $\mathrm{N}$-acyl group appears to slightly decrease the retention on PGC. In the middle trace, a tendency to separate the isomers with Neu5Gc on either the lower or upper arm can be seen. The nature of the peak at 57.5 remained unexplained.

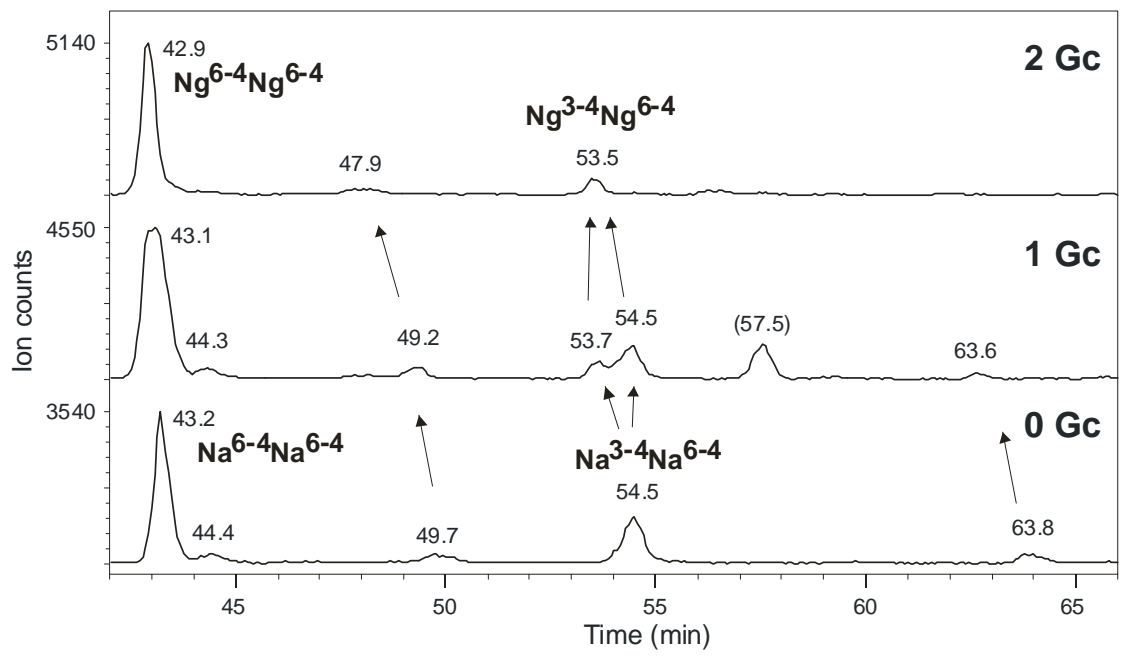


Figure S-5. Separation of selected structural features by PGC-LC. Panel A shows a chromatogram of a mixture of the triantennary $N$-glycans $\left[\mathrm{A}^{4} \mathrm{~A}^{4}\right] \mathrm{A}^{4}$ and $\mathrm{A}^{4}\left[\mathrm{~A}^{4} \mathrm{~A}^{4}\right]$. Panel $\mathbf{B}$ shows the LC-MS trace of three $\mathrm{N}$-glycans containing each three mannose and five GlcNAc residues. Panel $\mathbf{C}$ demonstrates the wide separation of the triantennary $N$-glycan $\mathrm{A}^{4}\left[\mathrm{~A}^{4} \mathrm{~A}^{4}\right]$ with either core $\alpha 1,6$-fucose or a Lewis $X$ fucose on the 4-antenna and in addition the peak of $\left[A^{4} A^{4}\right] A^{4} F^{6}$. Triangles stand for fucose residues, other symbols are as in Fig. S-1.

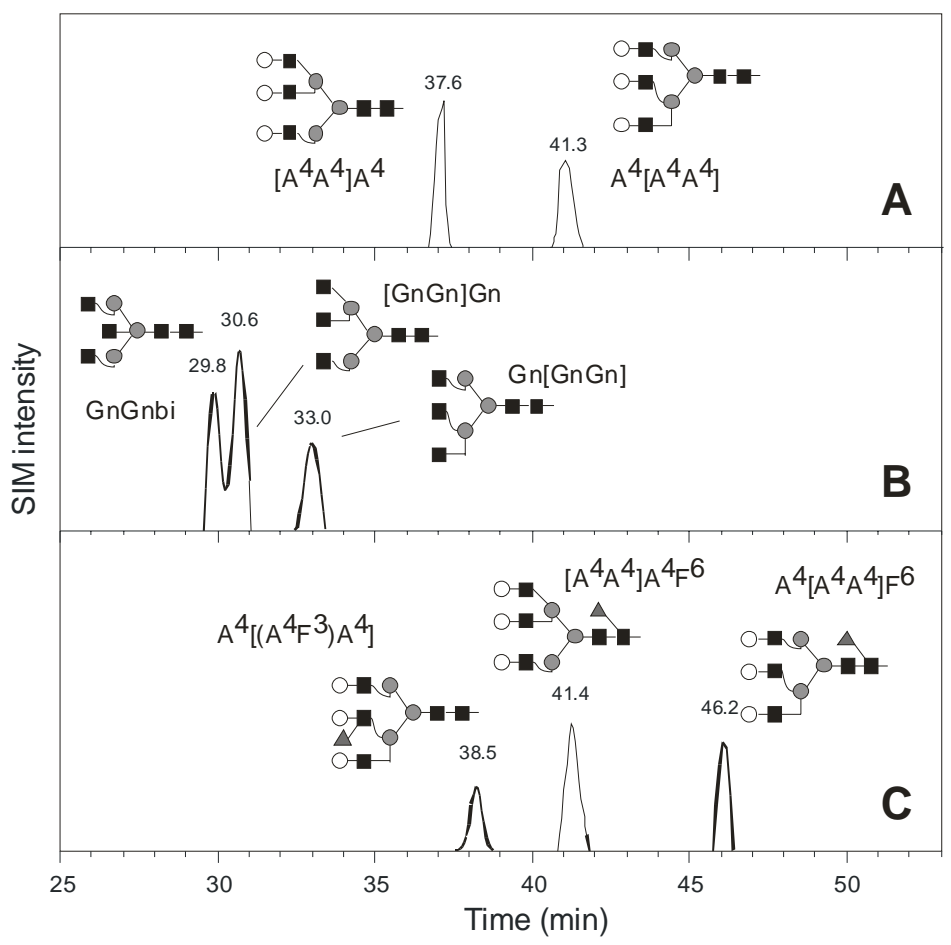

Figure S-6. In-source fragmentation. The chromatographic separation of very simple mixtures allows to acquire spectra of one particular glycan species. All ions can therefore be assigned as in-source fragments of this compound. Knowledge of these "MS ${ }^{1.5}$ " spectra prevents the erroneous detection of smaller oligosaccharides actually not being present in the sample. Panel A shows the spectrum of a $\mathrm{Na}^{6-4} \mathrm{Na}^{6-4}$ peak and panel $\mathbf{B}$ that of a GnGn peak. The loss of GlcNAc (Gn), Sia and Man residues

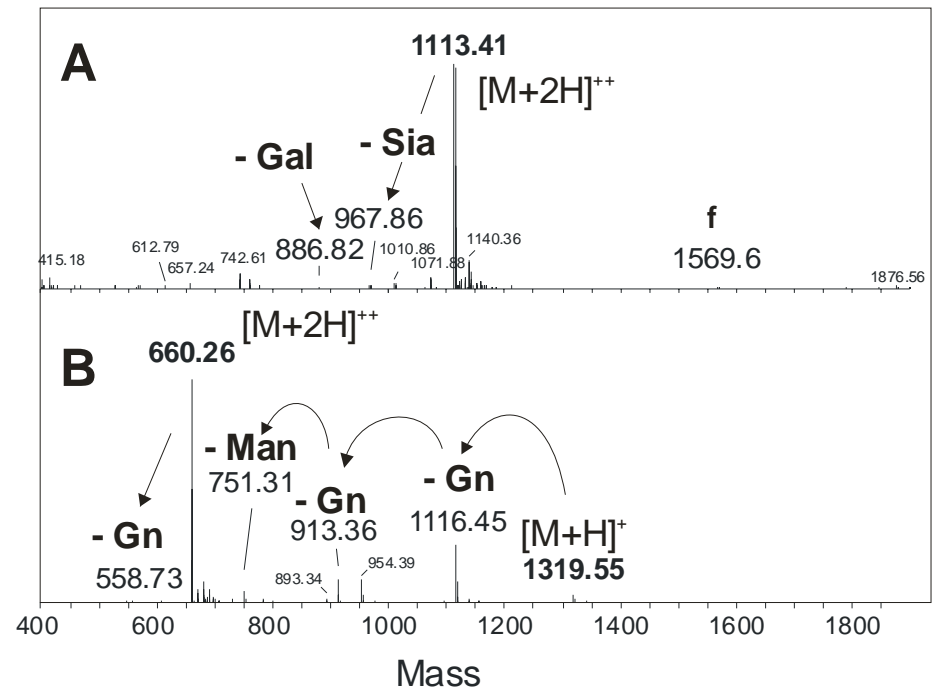
is indicated. The tiny peak labelled "f" in panel A results from the loss of an entire Neu5Ac-Gal-GlcNAc unit. Notably, the smaller oligosaccharide exhibited a much stronger in-source fragmentation than the larger, sialylated glycan. 
Table S-1. Summary of the fibrin(ogen) glycan structures. Only the number of sialic acids, of antennae (as Nacetyllactosamine-units $=\mathrm{Ln}$ ) and of fucose residues are considered here. What should be added is N-glycans of Chinese Hamster fibrin contained traces of triantennary, trisialylated structures (3S 3Ln) and were highly Oacetylated (36 \% mono-, $22 \%$ di-acetylated).

\begin{tabular}{|c|c|c|c|c|c|c|}
\hline \multirow[b]{2}{*}{ Fibrin from } & \multicolumn{6}{|c|}{ Overall composition of glycans } \\
\hline & 0S 2Ln & 1S 2Ln & 1S 2Ln 1F & $2 S 2 L n$ & $2 S$ 2Ln 1F & $3 S 2 \mathrm{Ln}$ \\
\hline & \multicolumn{6}{|c|}{ relative amount (\%) } \\
\hline Human & & 70.9 & & 29.1 & & \\
\hline Cow & 0.3 & 15.0 & & 84.5 & 0.2 & 0.9 \\
\hline Chicken & & 81.7 & & 18.3 & & \\
\hline Pig & & & 34.6 & & 65.4 & \\
\hline Mouse & & 7.6 & & 92.4 & & 4.0 \\
\hline Rat & & 0.7 & & 99.3 & & \\
\hline Cat & & 7.7 & & 92.3 & & \\
\hline Dog & & 36.7 & & 63.3 & & \\
\hline Chinese Hamster & & 11.5 & & 85.6 & 0.7 & 2.2 \\
\hline Horse & & 51.7 & & 48.3 & & \\
\hline Sheep & & 32.9 & & 64.3 & & 2.8 \\
\hline hum. transferrin & 0.8 & 21.2 & & 78.1 & & \\
\hline
\end{tabular}

\section{References for Supporting Information}

(1) Kolarich, D.; Weber, A.; Turecek, P. L.; Schwarz, H. P.; Altmann, F. Proteomics 2006, 6, 3369-3380.

(2) Kolarich, D.; Altmann, F. Anal Biochem 2000, 285, 64-75.

(3) Packer, N. H.; Lawson, M. A.; Jardine, D. R.; Redmond, J. W. Glycoconj J 1998, 15, 737-747.

(4) Wheeler, S. F.; Harvey, D. J. Anal Biochem 2001, 296, 92-100.

(5) Altmann, F.; Schweiszer, S.; Weber, C. Glycoconj J 1995, 12, 84-93.

(6) Strasser, R.; Stadlmann, J.; Svoboda, B.; Altmann, F.; Glossl, J.; Mach, L. Biochem J 2005, 387, $385-391$.

(7) Hennet, T.; Dinter, A.; Kuhnert, P.; Mattu, T. S.; Rudd, P. M.; Berger, E. G. J Biol Chem 1998, 273, 58-65.

(8) Sarkar, M.; Pagny, S.; Unligil, U.; Joziasse, D.; Mucha, J.; Glossl, J.; Schachter, H. Glycoconj J 1998, 15, $193-197$.

(9) Bencur, P.; Steinkellner, H.; Svoboda, B.; Mucha, J.; Strasser, R.; Kolarich, D.; Hann, S.; Kollensperger, G.; Glossl, J.; Altmann, F.; Mach, L. Biochem J 2005, 388, 515-525.

(10) Leiter, H.; Mucha, J.; Staudacher, E.; Grimm, R.; Glossl, J.; Altmann, F. J Biol Chem 1999, $274,21830-21839$.

(11) Zeleny, R.; Altmann, F.; Praznik, W. Anal Biochem 1997, 246, 96-101.

(12) Altmann, F.; Kornfeld, G.; Dalik, T.; Staudacher, E.; Gloss1, J. Glycobiology 1993, 3, 619-625.

(13) Tomiya, N.; Lee, Y. C.; Yoshida, T.; Wada, Y.; Awaya, J.; Kurono, M.; Takahashi, N. Anal Biochem 1991, 193, 90-100.

(14) Itoh, S.; Kawasaki, N.; Hashii, N.; Harazono, A.; Matsuishi, Y.; Hayakawa, T.; Kawanishi, T. J Chromatogr A 2006, 1103, 296306.

(15) Kawasaki, N.; Itoh, S.; Ohta, M.; Hayakawa, T. Anal Biochem 2003, 316, 15-22.

(16) Karlsson, N. G.; Wilson, N. L.; Wirth, H. J.; Dawes, P.; Joshi, H.; Packer, N. H. Rapid Commun Mass Spectrom 2004, 18, 22822292.

(17) Green, E. D.; Adelt, G.; Baenziger, J. U.; Wilson, S.; Van Halbeek, H. J Biol Chem 1988, 263, 18253-18268.

(18) Hokke, C. H.; Bergwerff, A. A.; Van Dedem, G. W.; Kamerling, J. P.; Vliegenthart, J. F. Eur J Biochem 1995, $228,981-1008$.

(19) Kawasaki, N.; Ohta, M.; Hyuga, S.; Hyuga, M.; Hayakawa, T. Anal Biochem 2000, 285, 82-91.

(20) Ninonuevo, M.; An, H.; Yin, H.; Killeen, K.; Grimm, R.; Ward, R.; German, B.; Lebrilla, C. Electrophoresis 2005, 26, 36413649 .

(21) Wilson, I. B. Glycoconj J 2001, 18, 439-447.

(22) Joziasse, D. H.; Schiphorst, W. E.; Van den Eijnden, D. H.; Van Kuik, J. A.; Van Halbeek, H.; Vliegenthart, J. F. J Biol Chem 1987, 262, 2025-2033.

(23) Weinstein, J.; de Souza-e-Silva, U.; Paulson, J. C. J Biol Chem 1982, 257, 13845-13853. 